\title{
AVALIAÇÃO DA QUALIDADE DE VIDA SEXUAL APÓS PRÁTICA DE GINÁSTICA FEMININA EM MULHERES JOVENS
}

\author{
Silvia Noffs,' Juliana Schulze Burti \\ EVALUATION OF SEXUAL QUALITY OF LIFE AFTER WOMEN GYMNASTICS \\ PRACTICE IN YOUNG WOMEN
EVALUACIÓN DE CALIDAD DE VIDA SEXUAL DE MUJERES JÓVENES TRAS PRACTICAR GIMNASIA PARA MUJERES

Resumo: A saúde sexual envolve aspectos físicos, emocionais e psicossociais. Para que esta seja plena e faça parte de uma vida com qualidade, todos os aspectos devem estar equilibrados. Muitos distúrbios sexuais podem envolver dificuldades ou disfunções pélvicas, como dor, fraqueza ou alterações de funcionalidade nos Músculos do Assoalho Pélvico (MAP). A literatura mostra que programas de treinamento dos MAP podem melhorar a função para resolução de distúrbios pélvicos, inclusive para melhora da sexualidade. Verificar a eficácia de um programa de exercícios para o assoalho pélvico, postura e consciência corporal, chamado ginástica feminina, na melhora da qualidade de vida sexual de mulheres jovens sexualmente ativas. A avaliação foi feita pelo Quociente de Sexualidade Feminino (QSF) validado para o português, comparando as respostas antes e depois da aplicação de cinco sessões semanais da ginástica feminina e também após seis meses do término do programa, verificando efeitos em curto e longo prazo. A prática de ginástica feminina promoveu melhora na qualidade de vida sexual das participantes em curto prazo, após cinco sessões, com melhora de 7,6\% no escore total do questionário. Melhores resultados foram encontrados nos aspectos conforto, excitação e relacionamento. Houve também melhora qualitativa, identificada por meio de relatos positivos das participantes, coletados no último encontro. Após seis meses do término, $66,6 \%$ das mulheres relataram continuar praticando os exercícios, com melhora na média do escore total, elevando a classificação de sexualidade para bom/excelente. A ginástica feminina se mostrou um método eficaz para promoção de saúde sexual em mulheres jovens em curto e longo prazo, além de ser acessível e de baixo custo. Os benefícios na sexualidade foram expressos principalmente pelo aumento do conforto e diminuição de dor durante o ato sexual, melhora da excitação e aumento da sintonia com a parceria.

Palavras-chave: Assoalho pélvico. Mulheres. Sexualidade.

\begin{abstract}
Sexual health involves physical, emotional and psychosocial aspects. To be satisfactory and part of a life with quality, all aspects must be balanced. A lot of sexual disorders can become from pelvic dysfunctions, such as pain, weakness, or functional disorders in the pelvic floor muscles (PFM). Literature shows that pelvic floor exercises programs can improve the function of pelvic disorders, including sexuality. Verify if an exercise program for PFM, posture and body awareness, called Women Gymnastics can increase the quality of sexual life of sexually active young women. The Female Sexuality Quotient (QSF), validated to Portuguese, was apllied before and after a five weeks program of Women Gymnastics, in order to compare some aspects of sexuality. To verify long term results, the QSF was apllied six months after the end of the program. The practice of Women Gymnastics improved $7.6 \%$ of QSF total score. Better results were observed in three spheres: comfort, arousal and relationship. There was also a quality improvement identified through positive reports. After six months $66 \%$ of women continued exercises practicing alone, with
\end{abstract}

\footnotetext{
Fisioterapeuta e docente de Educação Física, doutora em Psicologia Social pela Pontifícia Universidade Católica de São Paulo (PUC-SP), mestre em Ciências da Saúde pela Universidade Federal de São Paulo (Unifesp), docente e coordenadora de estágios do curso de Fisioterapia da PUC-SP. E-mail: juschulze@gmail.com

2 Doutora em Psicologia Social pela pontifícia Universidade Católica de São Paulo (PUC-SP). Mestre em Ciências da Saúde pela Universidade Federal de São Paulo (Unifesp). Especialista em Fisiologia do Exercício pela Unifesp. Aperfeiçoamento em Disfunções e Reeducação do Assoalho Pélvico pelo HSPESP. Professora do curso de Fisioterapia da PUC-SP. Autora do livro Ginástica Feminina: do assoalho pélvico à postura ideal. E-mail: silvianoffs@yahoo.com.br
} 
improvement of average total QSF score, raising the sexuality to good / excellent. Women Gymnastics is an effective method for promoting sexual health in young women in short and long term, and is useful and inexpensive. The benefits in sexuality were expressed in increased comfort and decreased pain during intercourse, increased arousal and better relationship with partner.

Keywords: Pelvic floor muscle. Woman. Sexuality.

Introducción: La salud sexual conlleva aspectos físicos, emocionales y psicosociales. Para que esta sea plena y brinde una buena calidad de vida, todos estos aspectos deben estar equilibrados. Varios trastornos sexuales pueden provenir de disfunciones pélvicas, como dolor, debilidad o trastornos funcionales en los músculos del piso pélvico (MPP). La bibliografía demuestra que los programas para ejercitar el piso pélvico pueden mejorar su función y ayudar a resolver los trastornos pélvicos e incluso mejorar la sexualidad. Verificar si un programa para ejercitar los MPP, la postura y la consciencia corporal, Ilamado Gimnasia Para Mujeres, puede aumentar la calidad de vida sexual de las mujeres jóvenes sexualmente activas. Se aplicó el Coeficiente Sexual Femenino (QSF), validado en portugués, antes y después de un programa de cinco sesiones semanales de Gimnasia Para Mujeres, con el objetivo de comparar algunos aspectos de la sexualidad. Para verificar sus resultados a largo plazo, el QSF se aplicó seis meses después de terminado el programa. La práctica de Gimnasia Para Mujeres resultó en el aumento en un 7,6 \% de los puntos totales de QSF. Se observaron mejores resultados en tres áreas: comodidad, deseo sexual y relacionamiento. Además, hubo una mejora cualitativa identificada a través de informes positivos. Luego de seis meses, el $66 \%$ de las mujeres continuó realizando los ejercicios, lo que mejoró el promedio de puntos totales del QSF y elevó su nivel de sexualidad a bueno/excelente. La Gimnasia Para Mujeres es un método efectivo para promover la salud sexual en mujeres jóvenes a corto y largo plazo; además, es accesible y de bajo costo. Los beneficios en cuanto a la sexualidad se observaron en el aumento de la comodidad y la disminución del dolor durante el coito, en el aumento del deseo sexual y en un mejor relacionamiento con la pareja.

Palabras clave: Piso pélvico. Mujeres. Sexualidade.

\section{Introdução}

A sexualidade envolve diversos aspectos da formação do ser humano, desde a infância até a vida adulta, tais como: biológico, educacional, religioso, cultural, ético, social, político, legal e econômico (LARA, 2009). Todos esses são fatores que podem influenciar a forma como o indivíduo viverá sua sexualidade. A saúde sexual é um dos pilares da qualidade de vida, segundo a Organização Mundial da Saúde (2002), o que torna o tema relevante para pesquisas e práticas.

De acordo com Basson (2000), a vivência sexual é afetada quando há alteração em alguma fase do ciclo de resposta sexual por mais de três meses. Segundo um estudo realizado na população brasileira, cerca de $34 \%$ das mulheres apresentam disfunção orgástica e $28 \%$ falta de desejo sexual. Entre os homens a prevalência de disfunções sexuais varia de 26 a $35 \%$, com problemas como ejaculação precoce, por exemplo (ABDO, 2004).

Considerando o contexto biopsicossocial que envolve a sexualidade, dificilmente poderemos abordá-la de forma isolada, avaliando somente uma variável. Em alguns casos, distúrbios de sexualidade estão relacionados com alterações biológicas, como tensão muscular, infecções ou inflamações crônicas da região genital (WEINHARDT, 1996). Um estudo epidemiológico encontrou que 21 a
$28 \%$ de mulheres sexualmente ativas relatam desordem de excitação e deficiência de lubrificação (LEWIS, 20I0).

A falta de conhecimento da própria anatomia, tabus em relação ao corpo e ao autotoque são fatores que podem comprometer também a função muscular dos Músculos do Assoalho Pélvico (MAP), que tem relação direta com a sexualidade (KEGEL, 1952). Um estudo realizado com 177 mulheres no período pré-climatério demonstrou que aquelas com melhor função da MAP apresentam melhor função sexual em comparação com mulheres que têm baixo grau de força e que não conseguem manter a contração desses músculos, ou seja, não têm resistência muscular (SACOMORI, 20I5). Bø e outros (2000), num estudo randomizado controlado, realizado com 59 mulheres com incontinência urinária, demonstraram que $\circ$ treinamento dos MAP reduziu as queixas relacionadas à vida sexual das mulheres, além de promover melhora na qualidade de vida sexual.

Há na literatura escassez de trabalhos a respeito de função sexual em mulheres jovens saudáveis. Em um dos poucos estudos comparou-se o grau de força muscular do assoalho pélvico antes e após o Treinamento dos Músculos do Assoalho Pélvico (TMAP), relacionando esta variável com o grau de satisfação sexual em mulheres, mostrando que o TMAP, como estratégia para incremento 
da força muscular, é positivo na melhora do prazer sexual (RIBEIRO, 20I2).

O método escolhido para trabalhar com as mulheres desta pesquisa foi o da ginástica feminina, que tem como foco a propriocepção dos MAP, de forma isolada e associados a exercícios respiratórios, de equilíbrio, resistência, coordenação e controle postural. As mulheres que participaram dos encontros não foram avaliadas de forma individualizada, por meio de toque bidigital e avaliação visual do assoalho pélvico, conforme orientações dos guidelines para profissionais de reabilitação (ROGERS et al., 2018), já que o objetivo da pesquisa não foi realizar tratamento fisioterapêutico ou reabilitação. $O$ método foi desenvolvido por uma profissional com formação em Fisioterapia e Educação Física. A ideia de sistematizar os exercícios surgiu durante aulas ministradas para grupos de mulheres em busca de promoção de saúde, que não necessariamente apresentavam queixas de disfunções pélvicas, mas que desconheciam o assoalho pélvico, e, após fazerem os exercícios reportavam melhora da sexualidade e diminuição de sintomas como perdas urinárias, fecais ou de flatos. Há pouca informação sobre essa temática em nossa sociedade, tornando importantes iniciativas como as da presente pesquisa, de levar conhecimento à população.

Segundo a Resolução n 046/2002, do Conselho Federal de Educação Física (CONFEF), atividade física é definida como

[...] todo movimento corporal voluntário humano, que resulta num gasto energético acima dos níveis de repouso, caracterizado pela atividade do cotidiano e pelos exercícios físicos. Trata-se de comportamento inerente ao ser humano com características biológicas e socioculturais. No âmbito da Intervenção do Profissional de Educação Física, a atividade física compreende a totalidade de movimentos corporais, executados no contexto de diversas práticas [...].

As atividades chamadas publicamente de ginástica são um exemplo. A mesma resolução define exercício físico como

[...] sequência sistematizada de movimentos de diferentes segmentos corporais, executados de forma planejada, segundo um determinado objetivo a atingir. Uma das formas de atividade física planejada, estruturada, repetitiva, que objetiva $\circ$ desenvolvimento da aptidão física, do condicionamento físico, de habilidades motoras ou reabilitação orgânico funcional, definido de acordo com diagnóstico de necessidade ou carências específicas de seus praticantes, em contextos sociais diferenciados.

De acordo com essas definições o nome ginástica atribuído ao método está correto. A ginástica feminina pode, portanto, ser executada por profissionais de Educação Física, desde que estes tenham formação adequada e especializada para entender a biomecânica da pelve e suas relações, e que não executem técnicas de avaliação e tratamento para reabilitação, exercício exclusivo do profissional fisioterapeuta.

Os fisioterapeutas, que em sua formação aprendem biomecânica, fisiologia do exercício, cinesioterapia, saúde da mulher, entre outras disciplinas, e estão inseridos nas equipes dos Núcleos de Apoio à Saúde da Família (NASF) - criados em 2008 pelo Ministério da Saúde, com objetivo de consolidar a Atenção primária no Brasil - muitas vezes participam de grupos terapêuticos e podem trabalhar com o método de ginástica feminina para promoção de saúde. Em 2016, o Conselho Federal de Fisioterapia e Terapia Ocupacional (Coffito), seguindo as suas atribuições legais, e a fim de trazer orientações ao exercício profissional da Fisioterapia, publicou, no Diário Oficial da União do dia 3 de outubro, o Acórdão $n^{\circ} 497$, que reconhecia a atividade chamada de treinamento funcional como uma ferramenta para o desenvolvimento de capacidades e que, portanto, pode ser considerada como competência do profissional fisioterapeuta. A ginástica feminina, de forma semelhante, pode ser considerada um método de exercícios que visa o trabalho corporal de forma global, buscando o equilíbrio das estruturas musculares, a prevenção de lesões e a melhora do controle e desempenho motor. A cinesioterapia, uma das principais disciplinas estudadas pelos fisioterapeutas e aplicadas na prática clínica, possui os mesmos objetivos, possibilitando ao profissional da Fisioterapia utilizar a ginástica feminina como forma de promoção de saúde.

A finalidade do método ginástica feminina foi promover consciência corporal de forma integral (BURTI, 2019). A hipótese do presente estudo foi que, após cinco encontros de prática dos exercícios da ginástica feminina, acompanhados de rodas de conversas em grupo sobre sexualidade, as mulheres teriam uma melhora de qualidade de vida sexual, promovida pela consciência corporal e controle da musculatura pélvica.

\section{Método}

Esta pesquisa de natureza analítica e experimental foi devidamente cadastrada na Plataforma Brasil e encaminhada para o Comitê de Ética da Pontifícia Universidade Católica de São Paulo (PUC-SP). O estudo foi realizado em dois ambientes: grupo I, na Paróquia Maria Mãe da Igreja; e grupo 2, na Pontifícia Universidade Católica de São Paulo, campus Perdizes. A amostra foi composta por mulheres com idade entre 18 e 30 anos, com vida sexual ativa, recrutadas voluntariamente via cartazes e via internet, que assinaram o Termo de Consentimento Livre e Esclarecido.

Foi utilizado para avaliar as participantes antes e após os cinco encontros de ginástica feminina o Quociente Sexual - Versão Feminina (QS-F) (ABDO, 2009). O questionário 
possui questões sobre alguns domínios da sexualidade e o escore total gera uma classificação de desempenho sexual. Os domínios do QS-F avaliam todas as fases do ciclo de resposta sexual além de contemplar outros domínios relacionados à sexualidade feminina.

O fluxograma (Figura I) explica a alocação dos grupos I e 2 e a junção das mulheres de ambos os grupos que participaram dos encontros e estavam dentro dos critérios de inclusão, que compõem a amostra final. A amostra final foi de 15 mulheres, que foram avaliadas e reavaliadas por meio do QS-F. Tanto a avaliação como a reavaliação foram feitas pela mesma pesquisadora, aluna de graduação de Fisioterapia treinada, acompanhada de fisioterapeuta docente responsável, para pesquisa de iniciação científica. $O$ total de 15 mulheres é a soma das integrantes do grupo I e das integrantes do grupo 2 que estavam dentro dos critérios de inclusão. As mulheres que foram excluídas, por desistência ou por não estarem dentro dos critérios de inclusão, não entraram na análise estatística do presente estudo. A pesquisadora conduziu cinco encontros semanais com duração de uma hora cada. Em cada encontro foi dedicado um momento inicial para as participantes falarem sobre a vida sexual e a prática da ginástica feminina na vida diária, seguido da realização dos exercícios, contidos no livro Ginástica feminina: do assoalho pélvico à postura ideal (BURTI, 20I7). Ao final dos encontros elas receberam uma apostila com o conteúdo dos exercícios e as participantes foram orientadas a praticar as atividades em casa.

Após seis meses do final do programa e da reavaliação foi feito novo contato via aplicativo de conversa Whatsapp com as 15 participantes que compuseram a amostra final para que elas respondessem virtualmente o questionário QS-F e comentassem a respeito da continuidade da prática dos exercícios. O objetivo desta reavaliação foi verificar os efeitos da ginástica feminina em longo prazo.

Figura I - Fluxograma

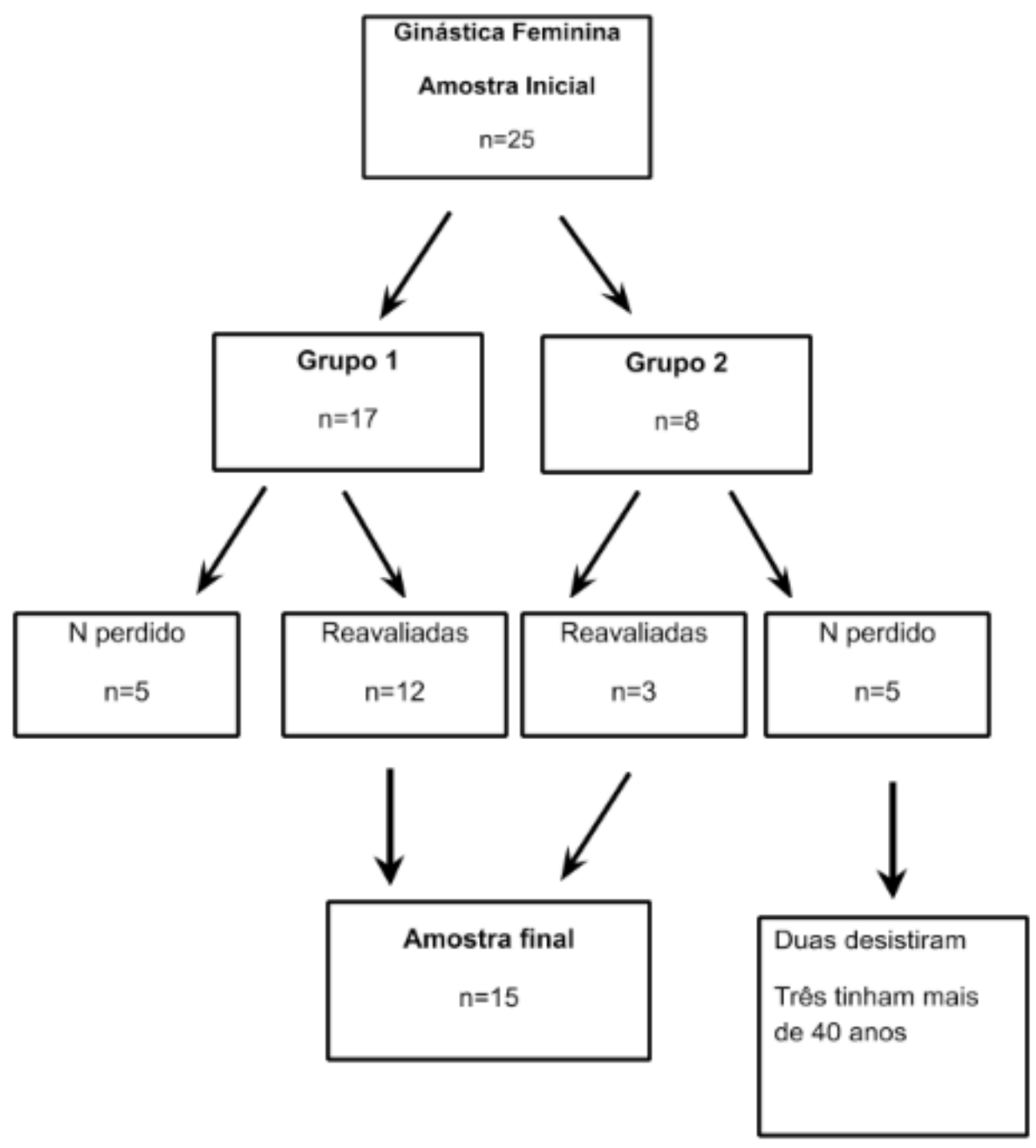




\section{Resultados}

A amostra inicial foi composta por 25 participantes voluntárias que assinaram o Termo de Consentimento Livre e Esclarecido sendo 17 no grupo I e oito no grupo 2. A média de idade foi de 24,3 anos (DP $\pm 5,8)$ e IMC de $24 \mathrm{~kg} / \mathrm{m}^{2}(\mathrm{DP} \pm 4,3)$. As mulheres da amostra tinham vida sexual ativa (100\%) e apenas $10 \%$ já tinham engravidado. Em relação à atividade física, $65 \%$ da amostra realizava algum tipo de atividade: $31 \%$ academia, $31 \%$ aula de dança, $15 \%$ ioga, $8 \%$ ciclismo, $8 \%$ pilates e $8 \%$ caminhada. Sobre problemas ginecológicos, que poderiam afetar os resultados, apenas $10 \%$ da amostra apresentava algum tipo de problema, sendo eles $50 \%$ cisto no ovário e $50 \%$ incontinência urinária. Para análise estatística foi utilizada o software Minitab versão I8. ANOVA I fator para comparação dos resultados antes e depois do tratamento. Foi adotado $p=0.05$ para nível de significância e foi realizada a porcentagem de melhora da amostra (Tabela I).

Tabela I - Porcentagem de melhora após cinco semanas de ginástica feminina e domínios do QS-F

\begin{tabular}{|c|c|c|c|}
\hline Variáveis & Pergunta & Domínios & $\begin{array}{l}\text { Porcentagem de } \\
\text { melhora da } I^{\mathrm{a}} \text { para } 2^{\mathrm{a}} \\
\text { avaliação }\end{array}$ \\
\hline Q I & $\begin{array}{l}\text { Você costuma pensar espontaneamente } \\
\text { em sexo, lembra de sexo ou se imagina } \\
\text { fazendo sexo? }\end{array}$ & Desejo e Interesse & $11,7 \%$ \\
\hline Q2 & $\begin{array}{l}\text { O seu interesse por sexo é suficiente } \\
\text { para você participar da relação sexual } \\
\text { com vontade? }\end{array}$ & Desejo e Interesse & $-6,7 \%$ \\
\hline Q3 & $\begin{array}{l}\text { As preliminares (carícias, beijos, abraços, } \\
\text { afagos etc.) a estimulam a continuar a } \\
\text { relação sexual? }\end{array}$ & Preliminares & $-4,2 \%$ \\
\hline Q4 & $\begin{array}{l}\text { Você costuma ficar lubrificada (molhada) } \\
\text { durante a relação sexual? }\end{array}$ & $\begin{array}{l}\text { Excitação pessoal } \\
\text { e sintonia com o } \\
\text { parceiro }\end{array}$ & $6,3 \%$ \\
\hline Q5 & $\begin{array}{l}\text { Durante a relação sexual, à medida } \\
\text { que a excitação do seu parceiro vai } \\
\text { aumentando, você também se sente mais } \\
\text { estimulada para o sexo? }\end{array}$ & $\begin{array}{l}\text { Excitação pessoal } \\
\text { e sintonia com o } \\
\text { parceiro }\end{array}$ & $11,1 \%$ \\
\hline Q6 & $\begin{array}{l}\text { Durante a relação sexual, você relaxa } \\
\text { a vagina o suficiente para facilitar a } \\
\text { penetração do pênis? }\end{array}$ & Conforto & $14,3 \%$ \\
\hline Q7 & $\begin{array}{l}\text { Você costuma sentir dor durante a } \\
\text { relação sexual, quando o pênis penetra } \\
\text { em sua vagina? }\end{array}$ & Conforto & $30,4 \%$ \\
\hline Q8 & $\begin{array}{l}\text { Você consegue se envolver, sem se } \\
\text { distrair (sem perder a concentração), } \\
\text { durante a relação sexual? }\end{array}$ & Desejo e interesse & I,9\% \\
\hline Q9 & $\begin{array}{l}\text { Você consegue atingir o orgasmo (prazer } \\
\text { máximo) nas relações sexuais que realiza? }\end{array}$ & $\begin{array}{l}\text { Orgasmo e } \\
\text { Satisfação }\end{array}$ & $23,5 \%$ \\
\hline Q10 & $\begin{array}{l}\text { O grau de satisfação que você consegue } \\
\text { com a relação sexual lhe dá vontade de } \\
\text { fazer sexo outras vezes, em outros dias? }\end{array}$ & $\begin{array}{l}\text { Orgasmo e } \\
\text { Satisfação }\end{array}$ & $12,8 \%$ \\
\hline $\begin{array}{l}\text { Escore } \\
\text { Total }\end{array}$ & & & $\begin{array}{l}(n=15) \\
7,6 \%\end{array}$ \\
\hline
\end{tabular}


Tabela 2 - Resultado da comparação entre os grupos

(continua)

\begin{tabular}{|c|c|c|c|c|c|c|}
\hline Variáveis & & Média & DP & IC & $\mathbf{F} ; \mathbf{p}^{*}$ & Melhora \\
\hline QI & $\begin{array}{l}\text { Pré }(n=20) \\
\text { Pós }(n=10) \\
\text { Follow up } \\
(n=10)\end{array}$ & $\begin{array}{l}3,7 \\
4,1 \\
3,8\end{array}$ & $\begin{array}{l}( \pm 0,9) \\
( \pm 1,2) \\
( \pm 0,8)\end{array}$ & $\begin{array}{l}{[3,3 ; 4,1]} \\
{[3,4 ; 4,8]} \\
{[3,1 ; 4,5]}\end{array}$ & $\begin{array}{l}I, 66 ; 0,2 I \\
0,40 ; 0,54\end{array}$ & $\begin{array}{l}11,7 \% \\
-7,3 \%\end{array}$ \\
\hline Q2 & $\begin{array}{l}\text { Pré } \\
\text { Pós } \\
\text { Follow up }\end{array}$ & $\begin{array}{l}4,5 \\
4,3 \\
4,3\end{array}$ & $\begin{array}{l}( \pm 0,9) \\
( \pm 1,3) \\
( \pm 0,8)\end{array}$ & $\begin{array}{l}{[3,9 ; 4,9]} \\
{[3,6 ; 5]} \\
{[3,5 ; 5,1]}\end{array}$ & $\begin{array}{l}0,37 ; 0,54 \\
0 ; 1\end{array}$ & $\begin{array}{l}-6,7 \% \\
0\end{array}$ \\
\hline Q3 & $\begin{array}{l}\text { Pré } \\
\text { Pós } \\
\text { Follow up }\end{array}$ & $\begin{array}{l}4,8 \\
4,7 \\
4,7\end{array}$ & $\begin{array}{l}( \pm 0,6) \\
( \pm 0,7) \\
( \pm 0,7)\end{array}$ & $\begin{array}{l}{[4,4 ; 5,1]} \\
{[4,2 ; 5,2]} \\
{[4,2 ; 5,2]}\end{array}$ & $\begin{array}{l}0,33 ; 0,57 \\
0 ; 1\end{array}$ & $\begin{array}{l}-4,2 \% \\
0\end{array}$ \\
\hline Q4 & $\begin{array}{l}\text { Pré } \\
\text { Pós } \\
\text { Follow up }\end{array}$ & $\begin{array}{l}3,7 \\
3,8 \\
4,5\end{array}$ & $\begin{array}{l}( \pm I) \\
( \pm I, 2) \\
( \pm 0,7)\end{array}$ & $\begin{array}{l}{[3,2 ; 4,2]} \\
{[3,1 ; 4,5]} \\
{[3,9 ; 5,1]}\end{array}$ & $\begin{array}{l}0,46 ; 0,5 \\
2,44 ; 0,14\end{array}$ & $\begin{array}{l}6,3 \% \\
18,4 \%\end{array}$ \\
\hline Q5 & $\begin{array}{l}\text { Pré } \\
\text { Pós } \\
\text { Follow up }\end{array}$ & $\begin{array}{l}3,9 \\
4,4 \\
4,2\end{array}$ & $\begin{array}{l}( \pm I, I) \\
( \pm 0,9) \\
( \pm 0,9)\end{array}$ & $\begin{array}{l}{[3,4 ; 4,4]} \\
{[3,8 ; 5,0]} \\
{[3,5 ; 4,9]}\end{array}$ & $\begin{array}{l}I, 03 ; 0,31 \\
0,23 ; 0,64\end{array}$ & $\begin{array}{l}11,1 \% \\
-4,5 \%\end{array}$ \\
\hline Q6 & $\begin{array}{l}\text { Pré } \\
\text { Pós } \\
\text { Follow up }\end{array}$ & $\begin{array}{l}3,5 \\
3,9 \\
4,4\end{array}$ & $\begin{array}{l}( \pm I, 3) \\
( \pm I, 7) \\
( \pm 0,7)\end{array}$ & $\begin{array}{l}{[2,9 ; 4,1]} \\
{[3,0 ; 4,8]} \\
{[3,6 ; 5,2]}\end{array}$ & $\begin{array}{l}I, I 6 ; 0,29 \\
0,72 ; 0,4 I\end{array}$ & $\begin{array}{l}14,3 \% \\
12,8 \%\end{array}$ \\
\hline Q7 & $\begin{array}{l}\text { Pré } \\
\text { Pós } \\
\text { Follow up }\end{array}$ & $\begin{array}{l}2,3 \\
1,6 \\
0,8\end{array}$ & $\begin{array}{l}( \pm I, 4) \\
( \pm I, 6) \\
( \pm 0,9)\end{array}$ & $\begin{array}{l}{[1,6 ; 2,9]} \\
{[0,7 ; 2,5]} \\
{[0,03 ; 1,1]}\end{array}$ & $\begin{array}{l}\text { I,89;0,18 } \\
I, 9 ; 0,18\end{array}$ & $\begin{array}{l}30,4 \% \\
50 \%\end{array}$ \\
\hline Q8 & $\begin{array}{l}\text { Pré } \\
\text { Pós } \\
\text { Follow up }\end{array}$ & $\begin{array}{l}3,6 \\
3,9 \\
4,3\end{array}$ & $\begin{array}{l}( \pm I) \\
( \pm I) \\
( \pm 0,8)\end{array}$ & $\begin{array}{l}{[3,1 ; 4,1]} \\
{[3,3 ; 4,5]} \\
{[3,7 ; 4,9]}\end{array}$ & $\begin{array}{l}0,04 ; 0,84 \\
0,96 ; 0,34\end{array}$ & $\begin{array}{l}1,9 \% \\
10,3 \%\end{array}$ \\
\hline Q9 & $\begin{array}{l}\text { Pré } \\
\text { Pós } \\
\text { Follow up }\end{array}$ & $\begin{array}{l}2,7 \\
3,1 \\
3,5\end{array}$ & $\begin{array}{l}( \pm I, 5) \\
( \pm I, 2) \\
( \pm I, I)\end{array}$ & $\begin{array}{l}{[2,0 ; 3,3]} \\
{[2,3 ; 3,9]} \\
{[2,8 ; 4,2]}\end{array}$ & $\begin{array}{l}2,13 ; 0,15 \\
0,62 ; 0,44\end{array}$ & $\begin{array}{l}23,5 \% \\
12,9 \%\end{array}$ \\
\hline
\end{tabular}


(conclusão)

\begin{tabular}{l|l|l|l|l|l|l} 
Variáveis & & Média & DP & IC & F\#; * & Melhora \\
\hline \multirow{2}{*}{ Q10 } & Pré & 3,9 & $( \pm I, 4)$ & {$[3,3 ; 4,5]$} & $I, 23 ; 0,27$ & $12,8 \%$ \\
& Pós & 4,4 & $( \pm I, 3)$ & {$[3,7 ; 5,0]$} & 0,$19 ; 0,67$ & $4,5 \%$ \\
& Follow up & 4,6 & $( \pm 0,7)$ & {$[3,9 ; 5,3]$} & & \\
\hline \multirow{2}{*}{ Total } & Pré & 74,2 & $( \pm I 5, I)$ & {$[66,5 ; 8 I, 9]$} & 0,$97 ; 0,33$ & $7,6 \%$ \\
& Pós & 80,2 & $( \pm 20,7)$ & {$[69,5 ; 90,9]$} & 0,$44 ; 0,5 I$ & $6 \%$ \\
& Follow up & 85 & $( \pm 9,7)$ & {$[74,5 ; 95,5]$} & & \\
\hline
\end{tabular}

O resultado negativo é referente à porcentagem de piora, ou seja, ao comparar a avaliação com a reavaliação houve uma diminuição na pontuação das questões dois e três e na questão um no follow-up após seis meses. Quando comparamos as médias dos escores inicial e final não houve diferença estatística significante, o que pode ter ocorrido devido ao tamanho pequeno da amostra, contudo, houve razão de significância entre os grupos na questão 8 , que trata de desejo e interesse. As questões Q6 e Q7, que tratam de conforto durante as relações sexuais, apresentaram, respectivamente, $14,3 \%$ e $30,4 \%$ de melhora, tendo em vista que a Q7 é calculada ao contrário, então embora a média tenha diminuído considera-se uma melhora de $30,4 \%$. No total, houve $7,6 \%$ de melhora no escore total em comparação ao grupo antes do treinamento, ou seja, apesar da melhora, o grupo continuou sendo classificado como regular a bom na sexualidade. A melhora do escore foi mais significativa nas participantes que tiveram uma primeira avaliação com escore baixo e apresentaram melhora gradual na segunda avaliação e no follow-up em comparação com a maioria das participantes, que tiveram um escore razoável na primeira avaliação.

Quadro 3 - Relatos escritos e domínios QS-F
$\mathrm{Na}$ reavaliação após seis meses, comparando as mulheres que responderam o questionário foi possível observar uma melhora na média do escore total, de 79,9 para 85, mudando a classificação da sexualidade de regular/bom para de bom/ótimo. Houve também uma melhora no resultado das médias das questóes seis e sete, relacionadas ao conforto durante $o$ ato sexual e na questão quatro, relacionada à excitação pessoal e sintonia com parceiro. As participantes que continuaram praticando podem ter aperfeiçoado o aprendizado e consciência, tanto com relação ao corpo como nos momentos de relacionamento.

Os resultados de melhora foram expressos também pelos relatos escritos das participantes ao final do último encontro, demonstrando que, embora a melhora quantitativa tenha sido modesta estatisticamente, houve melhora na qualidade da sexualidade.

Esses resultados podem ser vistos nos Quadros $3 \mathrm{e}$ 4, que trazem trechos dos relatos escritos associando-os com os domínios das questões do QS-F e os aspectos trabalhados durante os encontros da ginástica feminina, como consciência corporal e respiração.

\section{DOMÍNIOS DO QS-F RELATOS}

Desejo e interesse $\quad$ M., 22, Biomédica: "Após a prática dos exercícios percebi que o tesão e $a$ vontade de transar aumentaram. Além disso, percebi uma grande melhora na minha postura e na respiração."

Excitação Pessoal e sintonia com o parceiro(a)
J., 22, Estudante: "Embora eu não tenha tido relação sexual com alguém no período que fiz a ginástica, pude sentir uma grande diferença durante meu momento íntimo. Exercitar o assoalho pélvico trabalhando a respiração contribuiu para melhorar meu prazer." 


\section{DOMÍNIOS DO QS-F RELATOS}

\begin{tabular}{|l|l|}
\hline Conforto & $\begin{array}{l}\text { L., 22, Fisioterapeuta: “Depois que meu namoro com meu ex acabou me } \\
\text { sinto muito traumatizada e com medo de me relacionar com outras pessoas } \\
\text { apesar de estar em um novo relacionamento. A minha primeira vez não foi do } \\
\text { jeito que eu imaginei e senti muita dor e essa lembrança ficou, então sinto dor } \\
\text { em todas as relações devido a essa lembrança. Com os exercícios percebi que } a \\
\text { intensidade da dor diminuiu e a penetração foi mais facilitada comparando com as } \\
\text { outras vezes. Acho que se não tivesse uma questão emocional envolvida teria } \\
\text { percebido uma melhora maior.” }\end{array}$ \\
\hline Orgasmo e satisfação & $\begin{array}{l}\text { P., 24, Estudante: "A ginástica me possibilitou uma melhor consciência } \\
\text { corporal, que influenciou também durante o sexo, permitindo um maior } \\
\text { aproveitamento devido à propriocepção." }\end{array}$ \\
\hline
\end{tabular}

Quadro 4 - Relatos escritos e aspectos trabalhados na Ginástica Feminina

\begin{tabular}{|c|c|}
\hline Consciência corporal & $\begin{array}{l}\text { A., } 23 \text {, Estudante: "Senti uma pequena mudança, uma maior consciência } \\
\text { corporal e creio que com mais prática, mais frequente, os resultados } \\
\text { seriam notáveis." } \\
\text { M., 26, Psicóloga: "Os exercícios me ajudaram muito na consciência } \\
\text { corporal, mesmo em curto prazo e tenho certeza que em longo prazo os } \\
\text { resultados seriam ainda melhores." }\end{array}$ \\
\hline Post & $\begin{array}{l}\text { M., 22, Biomédica: " Após a prática dos exercícios percebi que o tesão } \\
\text { e a vontade de transar aumentaram . Além disso, percebi uma grande } \\
\text { melhora na minha postura e na respiração." }\end{array}$ \\
\hline $\begin{array}{l}\text { Treinamento dos músculos } \\
\text { do assoalho pélvico / } \\
\text { incontinência urinária }\end{array}$ & $\begin{array}{l}\text { J., 22, Estudante: "'Durante os encontros pude ter um controle melhor da } \\
\text { vagina com relação a segurar o xixi, pois tive enormes dificuldades para } \\
\text { segurar em situações comuns (tossir, espirrar) e após os encontros senti } \\
\text { uma enorme diferença." }\end{array}$ \\
\hline
\end{tabular}

\section{Discussão}

As participantes relataram que após a prática de ginástica feminina por cinco semanas elas tinham mais conhecimento do próprio corpo, não só em relação ao assoalho pélvico, mas também à postura e à respiração. A ginástica feminina aborda o TMAP e cria consciência da ação conjunta dos MAP com os estabilizadores de tronco e isso é feito em exercícios variados, incluindo movimentos para os membros superiores e inferiores, trabalhando o corpo todo e não isolando os MAP.

Ao realizar exercícios de tal maneira as participantes estavam trabalhando o corpo todo de forma funcional e isso proporcionou melhora na consciência corporal de forma geral, além de trabalhar músculos tônicos posturais, melhorando, também, a postura. $O$ grupo de músculos tônicos posturais é composto pelo diafragma, transverso do abdome, multífidos lombares e pelos músculos do assoalho pélvico. Todos os músculos são trabaIhados na ginástica feminina, através da respiração e da cocontração do transverso do abdome (BURTI, 20I7).
O trabalho dessa musculatura é benéfico para melhora da postura, pois são músculos que se inserem nas vertebras e na pelve e, quando suas características morfofuncionais são dentro do padrão de normalidade eles mantêm as curvaturas fisiológicas da coluna e o posicionamento adequado da pelve (NEUMANN, 2006; SANTOS, 20I I).

Essa melhora foi observada nos relatos escritos e ao longo dos encontros, já que esse domínio não é abordado no questionário. Algumas das falas que trazem esse aspecto: "Senti uma pequena mudança, uma maior consciência corporal e creio que com mais prática, mais frequente, os resultados seriam notáveis."; "Os exercícios me ajudaram muito na consciência corporal, mesmo em curto prazo e tenho certeza que em longo prazo os resultados seriam ainda melhores."; "[...] percebi uma grande melhora na minha postura e na respiração."

Sentir dor durante a penetração é algo que pode estar presente na vida sexual de muitas mulheres. Dados epidemiológicos mostram que 17,8\% das mulheres apresentam dispareunia - dor durante o ato sexual - e isso pode estar relacionado a fatores biopsicossociais, e à 
parceria sexual. Houve no presente estudo uma melhora de $30,4 \%$ no domínio relacionado ao conforto durante o ato sexual, mais especificamente à presença de dor durante a penetração.

A prática da ginástica feminina, mesmo com tempo de duração de cinco semanas, foi suficiente para que o conhecimento corporal e domínio da região pélvica trouxessem como benefício a diminuição da dor e maior conforto durante a penetração, pois o controle da musculatura do assoalho pélvico não ocorre apenas no momento da contração, mas também no relaxamento.

Praticar exercícios faz com que as mulheres percebam ambas as ações, contrair e relaxar, diferenciando-as e desenvolvendo melhor controle. Segundo Pereira-Baldon (2019), independente dos treinos serem feitos por pouco tempo e curta duração, se os exercícios forem feitos de forma correta são suficientes para proporcionar melhora na função do assoalho pélvico devido ao maior conhecimento e controle da musculatura, contudo, a frequência do treinamento é fundamental para manter a força e o controle dos MAP.

As participantes relataram melhora da consciência do assoalho pélvico através de seu treinamento e relataram que ter consciência e controle dessa musculatura aumentou $\circ$ prazer durante $\circ$ ato sexual. Em alguns relatos é possível identificar tal relação: "Após a prática dos exercícios percebi que o tesão e a vontade de transar aumentaram"; "Exercitar o assoalho pélvico trabalhando a respiração contribuiu para melhorar meu prazer".

As questões Q6, Q7, Q8, e Q9 do QSF, que são relacionadas com conforto e orgasmo, podem ter tido interferência de alguma resposta muscular obtida através do TMAP e da consciência corporal que a ginástica feminina promove.

Martinez e outros (2014) avaliaram 40 mulheres para investigar a relação do grau de força muscular dos MAP com uma boa função sexual. Verificaram uma relação entre mulheres com um alto grau de força muscular e um escore alto no questionário Female Sexual Function Index (FSFI), sugerindo que mulheres com força do assoalho pélvico e controle da musculatura tem uma melhor função sexual.

Um estudo que realizou o TMAP com 25 mulheres entre 18 a 40 anos com disfunção sexual por um período de dez sessões de uma a duas vezes por semana, comprovou que o TMAP foi positivo para redução das queixas e uma melhora na qualidade de vida sexual das mulheres estudadas, sendo que os autores afirmam que a conscientização da região pélvica junto com o aumento de força da musculatura pélvica promove melhora na função sexual (PIASSAROLLI et al., 20I0).

Apesar do tempo de treinamento do presente estudo ter sido menor comparado com Piassarolli e outros (2010), o resultado de melhora na conscientização e melhora na função foi semelhante, demostrando que fatores além do tempo de treinamento também podem influenciar os resultados. Dentre esses fatores estão I) promoção de educação, oferecendo conhecimento para as mulheres sobre a função do assoalho pélvico; e 2) frequência e adesão ao treinamento, ou seja, o quanto elas reproduziam o que aprendiam e o quanto treinavam sozinhas seguindo as orientações recebidas nos encontros. O ideal é que outros estudos sejam realizados com maior número de participantes e que levem em conta outras variáveis para avaliar qual tipo de TMAP é mais adequado para que haja mais resultados positivos na melhora da vida sexual de mulheres.

Uma revisão de literatura buscou a relação entre o TMAP e a função sexual feminina verificando que o treinamento promove aumento do fluxo sanguíneo para a pelve o que gera um aumento da sensibilidade no clitóris, e que consequentemente melhora as fases de excitação, lubrificação e orgasmo (FERREIRA, 20I5).

Uma limitação do presente estudo foi o número de participantes que realizaram a ginástica feminina. Um número maior de participantes em futuros estudos é necessário para avaliar de fato os efeitos da ginástica feminina na melhora da qualidade de vida sexual de mulheres jovens, contudo, os resultados apresentados foram satisfatórios do ponto de vista qualitativo, pois o conhecimento da função do assoalho pélvico integrado com a prática de atividade física com o TMAP promoveu melhora na qualidade de vida sexual das participantes. Outra limitação foi a ausência de grupo controle, o que deve ser incluído nos próximos estudos.

Para que haja alterações morfofuncionais nos músculos pélvicos (que são do tipo estriado esquelético), como aumento de força muscular, por exemplo, é necessário no mínimo três meses de treinamento, segundo o American College of Sports Medicine (1990), mas o objetivo do estudo não foi mensurar o grau de força muscular antes e após a ginástica feminina, mas sim avaliar a melhora na qualidade de vida sexual através da conscientização dos MAP.

\section{Conclusão}

Um programa de exercícios para postura, consciência corporal e treinamento dos MAP, a ginástica feminina, pode promover melhora na qualidade de vida sexual de mulheres jovens, em curto e longo prazo, tanto pelo treinamento dos músculos do assoalho pélvico e consciência corporal, como pelo espaço criado nos encontros para as mulheres falarem a respeito das próprias experiências e percepções sobre sexualidade e vivência sexual.

Os benefícios na sexualidade foram expressos pelo aumento do conforto e diminuição da dor durante $o$ ato sexual, melhora da excitação e aumento da sintonia com a parceria. Possivelmente com um maior tempo de treinamento os resultados seriam ainda mais positivos no âmbito 
de controle motor da musculatura do assoalho pélvico e de conscientização sobre saúde sexual e sexualidade. São necessários estudos randomizados e controlados com um número maior de participantes para comprovar os benefícios da ginástica feminina na sexualidade de mulheres jovens e saudáveis.

\section{Referências}

ABDO, C. H. N. et al. Estudo da vida sexual do brasileiro. São Paulo: Bregantini; 2004. 210 p. 3.

ABDO, C. H. N. Quociente sexual feminino: um questionário brasileiro para avaliar a atividade sexual da mulher. Diagnóstico e Tratamento, São Paulo, v. I4, n. 2, p. 89-91, 2009. Disponível em: http://files.bvs.br/upload/S/I4 I3-9979/2009/ vI4n2/a00I3.pdf

AMERICAN COLLEGE OF SPORTS MEDICINE. Position Stand/ The Recommended of Quantity and Quality of exercise for developing and maintaining cardiorespiratory and muscular fitness in healthy adults. Medicine and Science in Sports and Exercise, 1990.

BASSON, R. et al. Report of the international consensus development conference on female sexual dysfunction: definitions and classifications. The Journal of Urolology. v. 163, n. 3, p. 888893, Mar. 2000. Disponível em: https://www. sciencedirect.com/science/article/abs/pii/ S0022534705678287

BURTI, J. Ginástica feminina: do assoalho pélvico à postura ideal. Curitiba: Editora CRV, 2017.

BURTI, J. et al. Efeitos de exercícios para assoalho pélvico em mulheres idosas de diferentes níveis socioeconômicos. Revista Saúde e Pesquisa, v. 12, n. I, p. 39-49, jan./abr. 2019. Disponível em: https://periodicos.unicesumar. edu.br/index.php/saudpesq/article/view/6806

BØ, K.; TALSETH, T.; VINSNES, A. Randomized controlled trial on the effect of pelvic floor muscle training on quality of life and sexual problems in genuine stress incontinent women. Acta Obstetricia et Gynecologica Scandinavica, v. 79, n. 7, p. 598-603, 2000.

\section{CONSELHO FEDERAL DE EDUCAÇÃO FÍSICA} (Brasil). Resolução n. 046/2002. Dispõe sobre a Intervenção do Profissional de Educação Física e respectivas competências e define os seus campos de atuação profissional. Rio de Janeiro: Confef, 2002. Disponível em: https://www.confef. org.br/confef/resolucoes/82

\section{CONSELHO FEDERAL DE FISIOTERAPIA E TERAPIA OCUPACIONAL (Brasil). Acórdão $n^{\circ}$} 497, de 30 de setembro de 2016. O treinamento funcional é reconhecido como uma ferramenta para desenvolvimento de capacidades, podendo, portanto, ser considerado como uma competência do profissional fisioterapeuta. Brasília, DF: Conffito, 2016. Disponível em: https://www.coffito.gov.br/nsite/?p=5259

FERREIRA, C. et al. Does pelvic floor muscle training improve female sexual function? A systematic review. The international Urogynecological Association, v. 26, n. 12, p. 1735-1750, Dec. 2015. Disponível em: https:// link.springer.com/article/ I0.1007/s00192-0152749-y

GALDINO, L. A. C. S.; VARISE, E. M. Os efeitos do comando verbal na reabilitação física. Revista de Neurociencias, v. I8, n. I, p. 95-102, 2010.

KEGEL, A. Sexual functions of the pubococcygeus muscle. Western Journal of Surgery, Obstetrics, and Gynecology, Estados Unidos, v. 60, n. 10, p. $521-524,1952$.

LARA, L. Sexualidade, saúde sexual e Medicina sexual: panorama atual. Revista Brasileira Ginecologia e Obstetrícia, Rio de Janeiro, v. 31, n. I 2, p. 583-585, dez. 2009. Disponível em: https://www.scielo.br/scielo.php?pid=SOI00$7203200900|20000| \&$ script $=$ sci_arttext

LEWIS, R. W. et al. Definitions/epidemiology/risk factors for sexual dysfunction. The Journal of Sexual Medicine, v. 7, n. 4 (pt 2), p. 1598-1607, Apr. 2010. Disponível em: https://www.jsm.jsexmed.org/ article/SI 743-6095(I5)32996-9/fulltext

MARTINEZ, C. et al. Women with greater pelvic floor muscle strength have better sexual function. Acta Obstetricia et Gynecologica Scandinavica, v. 93, n. 5, p. 497-502, May 2014. Disponível em: https://obgyn.onlinelibrary.wiley.com/doi/ full/I0. I I I I/aogs. I 2379

NEUMANN, D. A. Cinesiologia do sistema musculoesquelético: fundamentos para reabilitação. Rio de Janeiro: Guanabara Koogan, 2006. 
ORGANIZAÇÃO MUNDIAL DA SAÚDE. Saúde reprodutiva e sexual: definição de saúde sexual. [Genebra?: OMS: 2002].

PEREIRA-BALDON, V. et al. Effects of different regimens for pelvic floor muscle training in young continent woman: Randomized controlled clinical trial. Journal of Eletromyograpy and Kinesiology, v. 44, p. 3I-35, Feb. 2019. Disponível em: https://www.sciencedirect.com/science/ article/abs/pii/S I 05064 I I | 8302530?via\%3Dihub

PIASSAROLLI, V. et al. Treinamento dos músculos do assoalho pélvico nas disfunções sexuais femininas. Revista Brasileira Ginecologia e Obstetrícia, Rio de Janeiro, v. 32, n. 5, p. 234-240, maio, 2010.

RIBEIRO, A.; ANKIER, S. A relação do grau de força muscular do assoalho pélvico com a satisfação sexual feminina. Revista Latinoamericana de Medicina Sexual - ReLAMS, São Paulo, v. I, n. I, 2012. Disponível em: https://www.slamsnet.org/ relams/pdf/relams-20I2-I-I4.pdf

ROGERS, R. G. et al. An International Urogynecological Association (IUGA)/International Continence Society (ICS) joint report on the terminology for the assessment of sexual health of women with pelvic floor dysfunction. Neurourology and Urodynamics. v. 37, n. 4, p. 1220-1240, Apr. 2018. Disponível em: https://onlinelibrary.wiley. com/doi/full/I0.1002/nau.23508
SACOMORI, C. et al. Pelvic floor muscle strength and sexual function in women. Fisioterapia em Movimento, Curitiba, v. 28, n. 4, p. 657-665, dez. 2015. Disponível em: http://www.scielo.br/scielo. php?script $=$ sci_arttext\&pid $=$ SO I 03-5 I $5020 \mid 500$ 0400657\&lng $=$ en\&nrm $=$ iso.

SANTOS, R. et al. Lumbar segmental stability. Medicina de Reabilitação, São Paulo, v. 30, n. I, p. I4-I7, jan./abr. 20II.

WEINHARDT L. S.; CAREY, M. P. Prevalence of erectile disorder among men with diabetes mellitus: comprehensive review, methodological critique, and suggestions for future research. The Journal of Sex Research, v. 33, n. 3, p. 205-213, 1996. Disponível em: https://www.tandfonline. com/doi/abs/I 0.1080/0022449960955 I836 